\title{
Hyaluronsäure- als Alternative zu Eigenserum- Augentropfen: Erste klinische Ergebnisse zu Augentropfen mit langkettiger Hyaluronsäure
}

\author{
Ria Beck ${ }^{a}$ Oliver Stachs ${ }^{a} \quad$ Anita Koschmieder ${ }^{a}$ Wolfgang G.K. Mueller-Lierheim ${ }^{b}$ \\ Sabine Peschel ${ }^{a}$ Gysbert-Botho van Setten ${ }^{c, d}$ \\ ${ }^{a}$ Augenklinik der Universität Rostock, Rostock, Deutschland; \\ ${ }^{b}$ i.com medical GmbH, München, Deutschland; \\ 'Augenklinik St. Erik, Karolinska Institutet, Stockholm, Schweden; \\ dInstitute of Wound Research and Department of Ophthalmology, University of Florida, Gainesville, FL, USA
}

\section{Schlüsselwörter}

Trockenes Auge · Eigenserum-Augentropfen · Sjögren-Syndrom . Wundheilung

\begin{abstract}
Zusammenfassung
Einleitung: Eigenserum-Augentropfen (ESAT) werden zur Behandlung des Trockenen Auges in besonders schweren Fällen eingesetzt. Nachdem mit ihrer Anwendung einmal begonnen wurde, gilt eine Rückkehr zu anderen gleitfördernden Augentropfen oder sonstigen Therapieoptionen auf dem Markt als unmöglich.

Material und Methoden: In einer randomisierten Studie wurde 11 Patienten, die seit mindestens 3 Monaten mit ESAT behandelt wurden, angeboten, stattdessen Augentropfen mit langkettiger Hyaluronsäure zu verwenden. Die Kontrollgruppe $(n=5)$ setzte ihre Behandlung mit ESAT fort. Die Verumgruppe $(n=6)$ wendete Augentropfen mit sehr langkettiger Hyaluronsäure (Comfort Shield ${ }^{\circledR}$ ) anstelle der ESAT an.
\end{abstract}

Ergebnisse: Von 4 der anfänglich 6 Patienten in der Verumgruppe, die die Studie abschlossen, entschieden sich 2 (50\%) dafür, auch über den Untersuchungszeitraum hinaus bei den Augentropfen mit langkettiger Hyaluronsäure zu bleiben, die anderen beiden kehrten zu ihrer vorherigen Therapie mit ESAT zurück. Die Kontrollgruppe führte ihre Behandlung fort wie zuvor und schloss die Studie nach 8 Wochen ab.

Schlussfolgerung: Erstmals erwiesen sich künstliche Augentropfen in Form von langkettigen Hyaluronsäure-Augentropfen als akzeptable Alternative zu ESAT. Einige Patienten empfanden diese Tropfen als noch besser als die Tropfen aus ihrem Eigenserum. Dies ist die erste Evidenz, dass durch Optimierung der Struktur des Hyaluronsäuremoleküls Augentropfen hergestellt werden könnten, die im Vergleich zu anderen Tränenersatzmitteln und sogar zu Eigenserum als besser wahrgenommen werden. Dies eröffnet neue Perspektiven für die Behandlung von Patienten mit schweren Fällen des Trockenen Auges.

(c) 2019 S.Karger GmbH, Freiburg

\section{Einleitung}

Eigenserum-Augentropfen (ESAT) stellen in schweren Fällen von Trockenem Auge eine wertvolle und gut verträgliche Behandlungsoption dar $[1,2]$. Sie erhalten häufig den Vorzug vor Tränenersatzmitteln [3], unter anderem weil sie eine gesunde
Oberfläche bzw. deren Wiederherstellung fördern [4] - ein Effekt, der dank eines stärker standardisierten Herstellungsprozesses auch besser vorhersagbar geworden ist [5-7]. Basierend auf der Konzentration biologischer Faktoren in normaler Tränenflüssigkeit [4] werden ESAT meist in 20\%-Serumkonzentration angewandt, wobei auch höhere Konzentrationen (von 50 bis information@karger.com

(c) 2020 S.Karger GmbH, Freiburg

www.karger.com/kop

Karger ${ }^{\prime}=$
Gysbert-Botho van Setten, MD, PhD, PhD

St Eriks Eye Hospital

Karolinska Institutet

Polhemsgatan 50, 11282 Stockholm, Schweden

gysbert-botho.vansetten@sankterik.se 
Tabelle 1. Anamnestische Merkmale der Patienten und deskriptive Daten der Verum- und Kontrollgruppe

\begin{tabular}{|c|c|c|c|c|c|}
\hline Patient & Abbruch & $\begin{array}{l}\text { Alter, } \\
\text { Jahre }\end{array}$ & Geschlecht & & $\begin{array}{l}\text { Dauer der ESAT-Vor- } \\
\text { behandlung, Monate }\end{array}$ \\
\hline \multicolumn{6}{|c|}{ Verum-Gruppe } \\
\hline 1 & & 79 & Männlich & Keratitis filiformis & 45 \\
\hline 2 & & 76 & Männlich & Allergische Konjunktivitis; Diabetes; adipohepatisch & 16 \\
\hline & $X$ & 32 & Männlich & Akute lymphatische Leukämie; Stammzelltransplantation & 82 \\
\hline 3 & & 80 & Weiblich & Neuropathische Keratopathie; Herpes zoster ophth. re.; Hornhautulkus; Katarakt & 45 \\
\hline & $x$ & 59 & Weiblich & Akute lymphatische Leukämie; Stammzelltransplantation & 20 \\
\hline 4 & & 67 & Weiblich & Sjögren-Syndrom & 50 \\
\hline \multicolumn{6}{|c|}{ Kontrollgruppe } \\
\hline 5 & & 72 & Weiblich & Keratokonjunktivitis sicca; Ptose und unvollständiger Lidschluss re. & 6 \\
\hline & $X$ & 88 & Weiblich & Keratokonjunctivitis sicca & 39 \\
\hline 6 & & 67 & Weiblich & Sjögren-Syndrom; Optikusatrophie re. & 39 \\
\hline 7 & & 82 & Weiblich & Keratokonjunktivitis sicca; Brennen; rheumatoide Arthritis & 13 \\
\hline 8 & & 59 & Weiblich & GVHD mit myelodysplastischem Syndrom; Stammzelltherapie & 10 \\
\hline
\end{tabular}

hin zu 100\%) zum Einsatz kommen [7-10]. Patienten, bei denen vier- bis achtmal täglich 20 - bis 50\%ige ESAT angewendet wurden, berichteten über eine subjektive Verbesserung der Symptome des Trockenen Auges; zugleich verzeichneten die jeweiligen Ärzte objektive Verbesserungen bei Parametern wie der Fluoreszeinfärbung oder der Tränenfilm-Aufrisszeit (BUT; break-up time) [16-23]. Der Gehalt an Zytokinen und weiteren Proteinen, die auch in der natürlichen Träne enthalten sind, macht ESAT zu einer so wertvollen Behandlungsoption [11]. Einige Zytokine, wie z.B. der epidermale Wachstumsfaktor, wurden in gesunder menschlicher Tränenflüssigkeit und im Serum in ähnlichen Konzentrationen nachgewiesen; TGF- $\beta$ und andere hingegen nicht $[3,12,13]$. Diese Beobachtung sprach für die Verwendung einer verdünnten Serumlösung mit einer ähnlichen TGF- $\beta$ Konzentration wie in Tränenflüssigkeit. Abweichungen in der Osmolarität des autologen Serums werden durch das Lösungsmittel, isotonische gepufferte Kochsalzlösung, ausgeglichen [14, 15]. Serumaugentropfen werden zur Behandlung von verschiedenen Schädigungen und Erkrankungen der Augenoberfläche eingesetzt; z.B. bei Tränenmangel im Zusammenhang mit dem Sjögren-Syndrom oder einer Graft-versus-Host-Reaktion, auBerdem bei neurotropher Keratopathie, persistierenden Epitheldefekten, oberer limbaler Keratokonjunktivitis oder Trockenem Auge nach einer LASIK-Operation. In allen diesen Settings haben Studienärzte über objektive Verbesserungen klinischer Parameter wie z.B. Fluoreszein-Färbung und BUT berichtet [1624]. Angesichts dieser bisher unerreichten Wirksamkeit wollten wir herausfinden, ob auch eine direkte Substitution der ESAT durch Hyaluronat eine Möglichkeit darstellt. Daher haben wir die hier beschriebene Untersuchung angestellt. Ermutigt durch die initialen Ergebnisse einer noch laufenden Studie zu Hyaluronsäure (Hyaluronan) mit langen Molekülketten haben wir in einer randomisierten Studie Patienten unter ESAT-Therapie diese Augentropfen als Alternative angeboten und die Wirksamkeit mit einer Kontrollgruppe verglichen, in der weiterhin ESAT angewandt wurden.

\section{Material und Methoden}

11 Patienten, die seit mindestens 3 Monaten mit ESAT behandelt wurden, erteilten nach Aufklärung ihre Einwilligung und wurden in die Studie aufgenommen und per Randomisierung in zwei Gruppen aufgeteilt. Für einen Zeitraum von 8 Wochen wurden in der Verumgruppe (6 Patienten) Augentropfen mit langkettiger Hyaluronsäure anstelle der ESAT angewandt, während in der Kontrollgruppe (5 Patienten) die ESAT-Therapie fortgeführt wurde (Tab. 1).

Bei drei Terminen (zu Studienbeginn, nach 4 Wochen und nach 8 Wochen) wurden anhand des OSDI-Fragebogens (Ocular Surface Disease Index) die Symptome beurteilt [27]. Die Anwendung der Tropfen (ad libitum) während der Studie wurde in Patientenprüfbögen erfasst.

\section{Eigenserum-Augentropfen}

Nach dem hochgradig standardisierten ESAT-Herstellungsverfahren an der Augenklinik der Universität Rostock wurden Vollblutproben in Serumröhrchen transferiert, 20 min bei Raumtemperatur stehengelassen und nach der Koagulation mit $2000 \mathrm{~g}$ bei $10^{\circ} \mathrm{C}$ für 20 min zentrifugiert. Aliquote wurden entnommen und durch $\mathrm{Zu}$ gabe von 0,9\%iger NaCl-Lösung (Balanced Salt Solution) im Verhältnis 1:1 verdünnt; anschließend wurden die Röhrchen verschlossen, mit einem Vortexer gemischt und bei $-18{ }^{\circ} \mathrm{C}$ eingefroren. Die Patienten nahmen ihre Röhrchen mit gefrorenem autologem Serum in einer Styroporbox mit nach Hause und lagerten sie bei $-18^{\circ} \mathrm{C}$. Erst unmittelbar vor Gebrauch tauten die Patienten jeweils ein Röhrchen auf und lagerten es bis zum Aufbrauchen gekühlt bei $2-8^{\circ} \mathrm{C}$.

\section{Hyaluronsäure-Augentropfen}

In dieser Studie wurden Augentropfen ohne Konservierungsmittel mit $0,15 \%$ iger sehr langkettiger Hyaluronsäure (Comfort Shield $^{\circledR}$, i.com medical GmbH, München, Deutschland) verwendet. Die Tropfen enthalten ausschließlich Stoffe, die auch natürlicherweise im menschlichen Auge vorkommen. 


\section{Diagnostik}

\section{Fluoreszein-Färbung der Cornea}

Hierzu wurde eine Natriumfluoreszein-Lösung ohne Konservierungsmittel verwendet (Fluorescéine Faure 0,5\% ophthalmische Lösung in 0,4-ml-Einheitsdosen, SERB SAS, Paris, Frankreich). Nach einem Lidschlag 20-120 s nach der Instillation wurden digitale Fotoaufnahmen der Augenoberfläche gemacht; die Kamera war eine Canon EOS 5D (Canon Inc., Tokio, Japan), angeschlossen an eine Takagi SM-70N Spaltlampe (Takagi Seiko Co., Ltd., Nagano, Japan). Die Auswertung des CFS (Corneal Fluorescein Staining) erfolgte nach der modifizierten Oxford-Skala [28]. Zusätzlich wurden die digitalen Fotografien zur automatisierten Beurteilung der Färbung elektronisch an das Auswertungszentrum der Freiburger Universitäts-Augenklinik übermittelt. Die Bilder wurden durch Anwendung adaptiver Schwellenwerte und automatisierte Berechnungen binarisiert; die resultierende Ratio galt als äquivalent mit dem Prozentsatz des sichtbaren Fluoreszeingefärbten Cornea-Areals. Aus diesem Verhältnis wurde der automatisierte Oxford-CFS-Grad berechnet [28].

\section{Weitere Untersuchungen}

Weitere Untersuchungen umfassten die Messung der TränenfilmBUT (Durchschnitt von drei Werten), Schirmer-I-Test ohne Anästhesie (Mark Blu Schirmer Tear Test Ophthalmic Strips; OptiTech Europe BVBA, Antwerpen, Belgien) sowie die Messung der Osmolarität der Tränenflüssigkeit mit dem Testsystem TearLab E-Series (TearLab Corporation, San Diego, CA, USA). Eine konfokale Laser-Scanning-Mikroskopie der Hornhaut in vivo wurde durchgeführt wie an anderer Stelle beschrieben $[29,30]$ unter Verwendung eines Heidelberg Retina Tomographen (HRT II, Heidelberg Engineering GmbH, Heidelberg, Deutschland) in Kombination mit dem an anderer Stelle beschriebenen Rostock Cornea Module [30]. Die subjektiven Symptome der Patienten wurden bei jedem Termin mit dem OSDI-Fragebogen erfasst. Auch die Anwendungshäufigkeit der Tropfen wurde ermittelt: Beim Studienbeginntermin wurden die Patienten befragt, wie oft sie ihre ESAT anwenden. Während der gesamten Studie wurde dann die Anwendung der Augentropfen (Hyaluronsäure oder Eigenserum) täglich protokolliert. Vor den diagnostischen Verfahren wendeten die Patienten mindestens 1 Stunde lang ihre Augentropfen nicht an.

\section{Statistik}

Aufgrund der begrenzten Anzahl teilnehmender Patienten wurde auf Signifikanzanalysen verzichtet, und eine deskriptive Analyse unter Vergleich von absoluten Zahlen und Mittelwerten wurde durchgeführt.

\section{Ergebnisse}

Von 11 Patienten, die initial in die Studie aufgenommen wurden, wurden 3 Patienten (einer aus der Kontrollgruppe, 2 aus der Verumgruppe) innerhalb des Untersuchungszeitraums vor dem planmäßigen Ende nach 8 Wochen ausgeschlossen; die Gründe
Tabelle 2. OSDI-Werte und Eintropfhäufigkeit in der Verum- und Kontrollgruppe

\begin{tabular}{|c|c|c|c|}
\hline Patient & Studienbeginn & 4 Wochen & 8 Wochen \\
\hline \multicolumn{4}{|c|}{ OSDI Verum } \\
\hline 1 & 71,9 & 44,4 & 41,7 \\
\hline 2 & 43,8 & 61,1 & 60,0 \\
\hline 3 & 65,6 & 67,5 & 72,5 \\
\hline 4 & 68,8 & 75,0 & 63,6 \\
\hline Mittel & 62,5 & 62,0 & 59,5 \\
\hline \multicolumn{4}{|c|}{ Eintropfhäufigkeit Verum } \\
\hline 1 & 14/Tag & 13/Tag & 13/Tag \\
\hline 2 & 14/Tag & 8/Tag & 10/Tag \\
\hline 3 & 6/Tag & 10/Tag & 8/Tag \\
\hline 4 & $14 /$ Tag & 18/Tag & 14/Tag \\
\hline Mittel & 12 & 12 & 11 \\
\hline \multicolumn{4}{|c|}{ OSDIKontrolle } \\
\hline 5 & 50,0 & 45,5 & 45,8 \\
\hline 6 & 52,3 & 47,7 & 50,0 \\
\hline 7 & 66,7 & 58,3 & 75,0 \\
\hline 8 & 42,5 & 62,5 & 57,5 \\
\hline Mittel & 52,9 & 53,5 & 57,1 \\
\hline \multicolumn{4}{|c|}{ Eintropfhäufigkeit Kontrolle } \\
\hline 5 & 14/Tag & 5/Tag & 5/Tag \\
\hline 6 & 28/Tag & 11/Tag & 11/Tag \\
\hline 7 & 14/Tag & 7,5/Tag & 7/Tag \\
\hline 8 & 7/Tag & 14/Tag & 15/Tag \\
\hline Mittel & 16 & 9,5 & 9,5 \\
\hline
\end{tabular}

waren Nichterscheinen, Demenz und Terminprobleme. 8 Patienten (4 in jeder Gruppe) nahmen weiter teil und absolvierten die Untersuchungen plangemäß (Tab. 1). Die Symptomatik laut durchschnittlichem OSDI-Score sowie die durchschnittliche Eintropfhäufigkeit veränderten sich während des 8-wöchigen Untersuchungszeitraums nicht signifikant (Tab. 2). Bei 2 Patienten in der Verumgruppe trat eine Verbesserung ein, die beiden anderen nahmen eine Verschlechterung der Symptome wahr.

Die OSDI-Werte der Patienten in der Verum- und der Kontrollgruppe korrelierten mit keinem der in der Studie betrachteten diagnostischen Parameter. Am Ende der Studie hatten sich bei 2 der 4 Verum-Patienten (Nr. 1 und 4), d.h. bei 50\%, die Symptome verbessert. Diese beiden Patienten entschieden sich dafür, auch weiterhin die Augentropfen mit langkettiger Hyaluronsäure anstelle der ESAT zu verwenden. Bei den anderen Patienten (Nr. 2 und 3) verschlechterten sich die Symptome. Diese beiden Patienten entschieden sich dafür, zur ESAT-Therapie zurückzukehren. Bei diesen beiden Patienten wurde festgestellt, dass die angebotene Behandlung ihnen keine signifikante Verbesserung brachte. Die Anwendungshäufigkeit der Hyaluronsäure-Augentropfen unterschied sich nur geringfügig von der ESAT-Anwendungshäufigkeit zum Zeitpunkt der Aufnahme in die Studie. In beiden Gruppen war im Verlauf der Studie ein geringfügiger Rückgang der protokollierten Eintropfhäufigkeit zu verzeichnen (Tab. 2). Die Ergebnisse der CFS-Tests sind in Tabelle 3 aufgeführt. Da die Augenoberflächen schwer geschädigt waren, waren die CFS-Ergebnisse in Teilen aufgrund von Unregelmäßigkeiten und Veränderungen der Oberflächen unzuverlässig. Diese Fälle wurden als nicht per Grading beurteilbar eingestuft
106

Kompass Ophthalmol 2020;6:104-110 DOI: $10.1159 / 000507412$ 
Table 3. CFS, BUT, Schirmer-I-Test und Osmolarität in der Verum- und Kontrollgruppe

\begin{tabular}{|c|c|c|c|c|c|c|c|}
\hline & \multirow[t]{2}{*}{ Patient } & \multicolumn{3}{|l|}{ Rechtes Auge } & \multicolumn{3}{|l|}{ Linkes Auge } \\
\hline & & Studienbeginn & 4 Wochen & 8 Wochen & Studienbeginn & 4 Wochen & 8 Wochen \\
\hline \multicolumn{8}{|c|}{ CFS-Test (Oxford-Skala) } \\
\hline \multirow[t]{4}{*}{ Verum } & 1 & III & IV & IV & I & IV & IV \\
\hline & 2 & k & k & k & III & k & k \\
\hline & 3 & IV & IV & k & III & IV & k \\
\hline & 4 & III & IV & III & । & III & I \\
\hline \multirow[t]{4}{*}{ Kontrolle } & 5 & k & IV & $\mathrm{k}$ & 0 & 0 & k \\
\hline & 6 & III & I & IV & IV & $\|$ & IV \\
\hline & 7 & 0 & I & I & 0 & I & । \\
\hline & 8 & IV & IV & k & IV & k & k \\
\hline \multicolumn{8}{|c|}{ TBUT-Test, s } \\
\hline \multirow[t]{4}{*}{ Verum } & 1 & 3 & ne & 2 & 3 & ne & 1 \\
\hline & 2 & 2 & 2 & 4 & 4 & 3 & 4 \\
\hline & 3 & 2 & 2 & 3 & 1 & 1 & 3 \\
\hline & 4 & 2 & 11 & 4 & 2 & 9 & 4 \\
\hline \multirow[t]{4}{*}{ Kontrolle } & 5 & 2 & 2 & 4 & 3 & 3 & 8 \\
\hline & 6 & 5 & 4 & 3 & 4 & 4 & 4 \\
\hline & 7 & 1 & 6 & ne & 1 & 12 & ne \\
\hline & 8 & 3 & 2 & 2 & 3 & 1 & 2 \\
\hline \multicolumn{8}{|c|}{ Schirmer-I-Test, $\mathrm{mm} / 5 \mathrm{~min}$} \\
\hline \multirow[t]{4}{*}{ Verum } & 1 & 1 & & 12 & 1 & & 17 \\
\hline & 2 & 1 & & 5 & 4 & & 9 \\
\hline & 3 & 0 & & 2 & 1 & & 0 \\
\hline & 4 & 5 & & 5 & 3 & & 6 \\
\hline \multirow[t]{4}{*}{ Kontrolle } & 5 & 0 & & 2 & 0 & & 0 \\
\hline & 6 & 1 & & 0 & 0 & & 0 \\
\hline & 7 & 1 & & 11 & 1 & & 14 \\
\hline & 8 & 0 & & 5 & 0 & & 7 \\
\hline \multicolumn{8}{|c|}{ Osmolarität, mosm/l } \\
\hline \multirow[t]{4}{*}{ Verum } & 1 & 291 & & 293 & 293 & & 286 \\
\hline & 2 & 287 & & 302 & 282 & & 290 \\
\hline & 3 & k & & 315 & 306 & & 311 \\
\hline & 4 & k & & 283 & k & & $<280$ \\
\hline \multirow[t]{4}{*}{ Kontrolle } & 5 & 300 & & k & 312 & & 306 \\
\hline & 6 & 308 & & 307 & 309 & & 302 \\
\hline & 7 & 318 & & k & 320 & & 306 \\
\hline & 8 & 284 & & 301 & k & & 295 \\
\hline
\end{tabular}

k: kein Grading möglich; ne: nicht ermittelt.

Ebenso war auf den Unregelmäßigkeiten aufweisenden, rauen Oberflächen eine zuverlässige Messung der Tränenfilm-BUT oft nicht möglich (Tab. 3). Der Schirmer-I-Test war etwas erfolgreicher; er ergab ein gewisses Maß an Verbesserung in beiden Gruppen mit lediglich einem kleinen Unterschied zwischen den Gruppen in Form geringfügig besserer Werte in der Verumgruppe (Tab. 3).

Die Messung der Osmolarität erwies sich bei diesen Patienten als echte Herausforderung, da der Tränenmeniskus in den meisten Augen minimal war. So konnte bei einem Teil der Patienten kein ausreichendes Volumen entnommen werden, und der Parameter musste als nicht messbar notiert werden. Interessanterweise wurde jedoch bei keinem Patienten eine Osmolarität von mehr als 320 mosm/l gemessen, und der Unterschied zwischen beiden Augen war minimal (Tab. 3).
Zum Zeitpunkt der Aufnahme in die Studie waren die subbasalen Nervenplexus der mit konfokaler Laser-Scanning-Mikroskopie untersuchten Patienten sehr heterogen. Zwischen den Patienten bestanden signifikante Unterschiede von einer kaum vorhandenen bis hin zu einer brauchbaren Innervation. Sowohl in der Verumgruppe (s. Abb. 1) als auch in der Kontrollgruppe lagen gut definierte subbasale Nerven vor. Hinsichtlich der Innervation und des Erscheinungsbilds des subbasalen Nervenplexus zu Studienbeginn und nach 8 Wochen waren keine größeren Unterschiede zu beobachten. Insgesamt erwies sich das Erscheinungsbild der Cornea auf Höhe des subbasalen Nervenplexus sowohl in der Verum- als auch in der Kontrollgruppe als extrem heterogen. Die diesbezüglichen Unterschiede korrelierten nicht mit anderen Zeichen und Symptomen. Auch hatten diese Unterschiede keine 


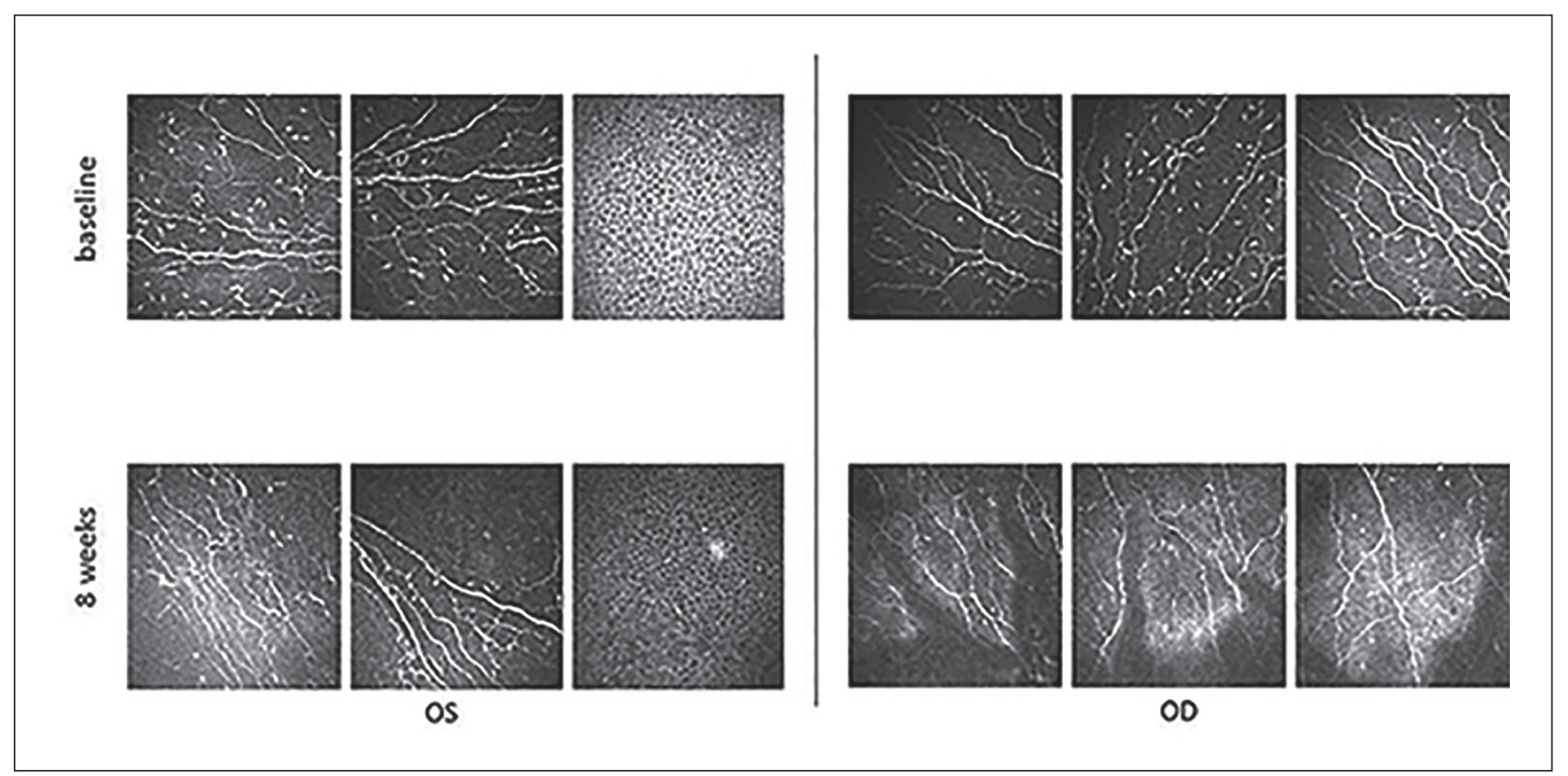

Abb. 1. Hornhautnerven eines Patienten aus der Verumgruppe, der auch nach der Studie bei den Hyaluronsäure-Augentropfen blieb.

prädiktive Aussagekraft hinsichtlich der Entscheidung der Patienten für oder gegen die weitere Anwendung der langkettigen Hyaluronsäure-Augentropfen auch nach dem Ende der Studie.

\section{Diskussion}

Dies ist unseres Wissens der erste Bericht, der belegt, dass Patienten künstliche Augentropfen gegenüber Produkten aus Eigenblut bevorzugen, welche bisher als höchste Stufe der fortgeschrittenen Behandlung schwerster Fälle des Trockenen Auges galten. In der hier vorgestellten Studie konnten wir zeigen, dass Augentropfen mit sehr langkettiger Hyaluronsäure von einem Teil der Patienten als bevorzugte Alternative zu ESAT wahrgenommen werden. Diese Studie zeigt auf, dass Hyaluronsäure allein, wenn sie in optimaler galenischer Formulierung, in der richtigen Konzentration und in einer Molekülgröße ähnlich der natürlicherweise an der Augenoberfläche vorkommenden Hyaluronsäure auf die erkrankte Augenoberfläche aufgebracht wird, für einen Teil der Patienten eine mögliche Alternative zur Anwendung von ESAT darstellt. Den Autoren sind keine weiteren Berichte bekannt, denen zufolge das letzte Mittel zur Behandlung schwerer Fälle von Trockenem Auge, also Serumaugentropfen, durch synthetische Tränenersatz-Augentropfen erfolgreich ersetzt werden konnte. In unserer Studie konnten wir zeigen, dass über einen Zeitraum von 8 Wochen 4 von 6 Patienten, die seit langem ESAT anwendeten, auf hochmolekulare Hyaluronsäure-Augentropfen umgestellt werden konnten, ohne dass sich die Parameter der Augenoberflächenerkrankung signifikant veränderten, und dass bei 2 dieser Patienten sogar eine Besserung der Symptome laut OSDI eintrat. 2 von 4 Patienten entschieden sich jedoch auch für die Rückkehr zur ESAT-Behandlung, nachdem sie 8 Wochen lang die Alterna- tivoption ausprobiert hatten. Bei diesen Patienten könnte möglicherweise eine Kombination aus Hyaluronsäure und Serumaugentropfen zu einer Kombination aus positiven Effekten im Rahmen einer allmählichen, langsamen Ausschüttung von Wachstumsfaktoren über einen längeren Zeitraum führen, was wiederum günstige Auswirkungen auf die Augenoberfläche haben könnte, wie eine kürzlich veröffentlichte Studie nahelegt [31]. Auch hier mussten ESAT aus dem Kühlschrank verwendet werden. Interessanterweise könnte jedoch bei dieser Kombination der Einsatz von langkettiger Hyaluronsäure die entzündungshemmende Wirkung der Serumaugentropfen verstärken, wie von Stenwall und Kollegen berichtet [11].

Die Anwendung von Augentropfen mit sehr langkettiger Hyaluronsäure anstelle von ESAT hat praktische Aspekte. Sie könnten nicht nur als vorübergehende Ergänzung zu oder Ersatz für Serumaugentropfen dienen, sondern könnten auch initial anstelle von Serumaugentropfen angewandt werden, wenn diese aus regulatorischen oder kulturellen Gründen keine Option sind oder wenn nur allogene Serumaugentropfen verfügbar sind. Darüber hinaus sind die Augentropfen mit sehr langkettiger Hyaluronsäure kein Blutprodukt und gehen daher mit keinem Infektionsrisiko einher. Sie erfordern keine besonderen Transport- und Lagerungsbedingungen wie Kühlen/Gefrieren und sind weniger kostspielig. Diese Studie unterstreicht die Bedeutung nicht nur der sehr langkettigen Hyaluronsäure, die in Comfort Shield ${ }^{\circledR}$-Augentropfen enthalten ist, und ihrer Konzentration, die das Fließverhalten gesunder menschlicher Tränenflüssigkeit imitiert, sondern dieser galenischen Form insgesamt. Diese Studie untermauert außerdem die wachsende Evidenz dafür, dass die Osmolarität kein geeignetes diagnostisches Instrument in schweren/sehr schweren Fällen von Trockenem Auge darstellt, da wir bei keiner Messung 
eine signifikante Hyperosmolarität feststellten (Höchstwert: 320 mosm/l). Dieses Ergebnis deckt sich mit einem früheren Bericht, der keinen statistischen Unterschied zwischen der Osmolarität der Tränenflüssigkeit von gesunden Probanden (306 mosm/l) und der von Patienten mit Trockenem Auge (302 und 301 mosm/l vor bzw. nach der Behandlung mit Serumaugentropfen) feststellte [32]. Die Anwendung isotonischer Augentropfen auf einer Augenoberfläche normaler Osmolarität vermeidet jeglichen provozierten osmotischen Stress durch Schwankungen der Osmolarität auf einer geschädigten und chronisch entzündeten Augenoberfläche. Dies ist von umso größerer Relevanz, da Hyperosmolarität (>308 mosm/l) als wichtigster kausaler Faktor in der Pathophysiologie des schweren Trockenen Auges und als der verursachende Faktor im Teufelskreis des Trockenen Auges benannt worden ist $[33,34]$. Aktuelle und zukünftige Studien werden weitere Evidenz zu dieser wichtigen Fragestellung liefern.

In der hier vorgestellten Studie lag bei keinem der anderen untersuchten Parameter des Trockenen Auges (Schirmer-Test, BUT etc.) ein signifikanter Unterschied zwischen der Verum- und der Kontrollgruppe vor. Darüber hinaus zeigte diese Studie, dass in sehr schweren Fällen von Trockenem Auge der Zustand der Oberfläche nicht immer eine zuverlässige Anwendung eines Augenoberflächen-Staining-Scores ermöglicht. Ähnlich diesen diagnostischen Schwierigkeiten bei schwerem Trockenen Auge gab auch die konfokale Mikroskopie des subbasalen Nervenplexus keinen Aufschluss darüber, ob ein Patient die Substitution der ESAT durch langkettige Hyaluronsäure-Augentropfen akzeptieren würde oder nicht.

Bei der Behandlung schwerer Erkrankungen der Augenoberfläche mit ESAT sollten wir uns bewusst sein, dass die Zusammensetzung des Serums bei multimorbiden Patienten durch den allgemeinen Gesundheitszustand wesentlich verändert sein kann. Sie ist zwar in Konzentration und Produktion weitgehend standardisiert, hängt jedoch von der Zusammensetzung des Blutes ab, die wiederum durch den konkreten Gesundheitszustand des Patienten bestimmt wird [3]. Grundsätzlich ist die Konzentration einiger Komponenten im Tränenfilm höher als im Serum, die von anderen (wie zum Beispiel Vitamin A) ist hingegen im Serum deutlich höher [35-37]. Bei systemischen Erkrankungen wie z.B. Bindegewebserkrankungen sind die Serumlipidprofile signifikant verändert [38]. Kardiovaskuläre Erkrankungen können die Konzentration von Plasminogenaktivatoren beeinflussen [39]. Bei Patienten mit rheumatoider Arthritis wurden erhöhte Konzentrationen von VEGF und IL-6 gemessen [40]. Und die IL-1 $\beta$ und TNFa-Konzentrationen sind im peripheren Blut von Patienten mit primärem Sjögren-Syndrom erhöht [41]. Wenn man all dies bedenkt und dazu berücksichtigt, dass diese Veränderungen je nach Schwere der Erkrankung unterschiedlich ausgeprägt sind und somit auch im Verlauf der Behandlung variieren, liegt es auf der Hand, dass die Qualität und Quantität verschiedener Faktoren im Serum variieren kann. Das bedeutet auch eine Variation der Wirksamkeit und Verträglichkeit von ESAT in schwer erkrankten Augen. Es ist nicht unwahrscheinlich, dass das Serum von Patienten mit höchster allgemeiner Krankheitsaktivität eine defizitäre und potenziell schädliche Zusammensetzung aufweist. Gerade hier bieten Augentropfen mit langkettiger Hyaluronsäure große Vorteile dank ihrer reproduzierbaren Zusammensetzung und Freiheit von potenziell schädlichen Inhaltsstoffen. Augentropfen mit langkettiger Hyaluronsäure bieten mit hoher Wahrscheinlichkeit signifikante Vorteile insbesondere für die Behandlung von schwerem Trockenen Auge bei Patienten mit schweren systemischen Erkrankungen. Hinzu kommt, dass bei schweren systemischen Erkrankungen oft keine guten Rahmenbedingungen für häufige und wiederholte Blutabnahmen herrschen.

Die in dieser Studie verwendeten Augentropfen mit langkettiger Hyaluronsäure haben ein bisher ungedecktes therapeutisches Potenzial und bieten zumindest vorübergehend eine wirksame Alternative zu ESAT. Dies gilt umso mehr, als dass manche Patientengruppen weniger gut auf Serumaugentropfen ansprechen. Gemäß Hwang et al. [42] sind ESAT möglicherweise nicht für die Anwendung beim sekundären Sjögren-Syndrom (wegen erhöhter Serumspiegel proinflammatorischer Zytokine) geeignet. Bei diesen Patienten könnten Augentropfen mit langkettiger Hyaluronsäure eine wertvolle Alternativoption darstellen. Jedoch liegen bisher keine spezifischen Leitlinien vor, um Patienten zu identifizieren, bei denen die Anwendbarkeit von ESAT wegen systemischer Erkrankung limitiert ist oder bei denen die Qualität der ESAT infolge der Art der zugrunde liegenden systemischen Erkrankung suboptimal ist. Diese Bedingungen würden für die Anwendung von Augentropfen mit langkettiger Hyaluronsäure sprechen.

\section{Schlussfolgerung}

Augentropfen mit langkettiger Hyaluronsäure zeichnen sich durch einzigartige Eigenschaften aus, die denen der natürlicherweise auf der Augenoberfläche vorkommenden Hyaluronsäure so ähnlich sind, dass ein Teil der Patienten diese Tropfen als noch besser zu empfinden scheint als Tropfen aus Eigenserum. Die Studie liefert klare Hinweise darauf, dass die Molekülstruktur und Kettenlänge der Hyaluronsäure von klinischer Relevanz sind. Dies ist die erste Evidenz, dass die Optimierung der Struktur des Hyaluronsäuremoleküls zu Augentropfen führen könnte, die in bisher unerreichtem Maße natürlicher Tränenflüssigkeit ähneln. Die geringe Fallzahl stellt indessen eine Beschränkung der Studie hinsichtlich der Ergebnisse und ihrer Interpretation dar. Um die hier präsentierten Ergebnisse weiter zu untermauern, sind weitere prospektive Studien mit größeren Teilnehmerzahlen und eventuell einem Studienaufbau mit Umstellung der Therapie erforderlich.

\section{Danksagungen}

Professor Dr. med. Daniel Böhringer, Augenklinik der Universität Freiburg, Deutschland, gilt großer Dank und Anerkennung für seinen bedeutenden Beitrag zur Entwicklung und Umsetzung der angewandten Methoden. Die Autoren danken der Forschungsstiftung SFF (Synfrämjandets Forskningsfond), Stockholm, Schweden, dass sie Zeit für die Durchführung dieser Studie gewährt hat. Der Aviation-Ophthalmology, Inc., Schweden gilt dankbare Anerkennung für die Bereitstellung von Know-how, technischer Hilfe und Unterstützung. 


\section{Ethikerklärung}

Die Studie wurde von der lokalen Ethikkommission sowie vom Bundesinstitut für Arzneimittel und Medizinprodukte (BfArM) genehmigt, ent spricht EN ISO 14155 [25] sowie der Deklaration von Helsinki und ist in der EUDAMED unter der Nummer CIV-16-06-015964 registriert. Als Sponsor und Monitor der Studie war die CORONIS GmbH (München, Deutschland) tätig, eine Organisation mit einem Qualitätsmanagementsystem für die klinische Forschung mit Zertifizierung und jährlicher $\mathrm{Au}$ ditierung nach der internationalen Norm EN ISO 13485 durch eine europäische Benannte Stelle [26].

\section{Disclosure Statement}

Ria Beck und Gysbert van Setten haben keine finanziellen Interessen in Verbindung mit dieser Publikation. Dr. Wolfgang Müller-Lierheim hat gleichzeitige Interessen als Sponsor der Studie in seiner Position als CEO der i.com medical GmbH, München, Deutschland.

\section{Finanzierungsquellen}

i.com medical GmbH, München, Deutschland.

\section{Literatur}

$\checkmark 1$ Fox RI, Chan R, Michelson JB, et al.: Beneficia effect of artificial tears made with autologous serum in patients with keratoconjunctivitis sicca. Arthritis Rheum. 1984;27:459-461.

$\checkmark 2$ Giannaccare G, Versura P, Buzzi M, et al.: Blood derived eye drops for the treatment of cornea and ocular surface diseases. Transfus Apheresis Sci. 2017;56:595-604.

3 Pan Q, Angelina A, Marrone M, et al.: Autologous serum eye drops for dry eye. Cochrane Database Syst Rev. 2017;2:CD009327.

$\checkmark 4$ Young AL, Cheng AC, Ng HK, et al.: The use of autologous serum tears in persistent corneal epithelial defects. Eye (Lond). 2004; 18: 609-614.

5 Geerling G, Unterlauft JD, Kasper K, et al. [Autologous serum and alternative blood products for the treatment of ocular surface disorders]. Ophthalmologe. 2008; 105 623-631.

6 Liu L, Hartwig D, Harloff S, et al.: An optimised protocol for the production of autologous serum eyedrops. Graefes Arch Clin Exp Ophthalmol. 200;243:706-714.

7 Geerling G, Maclennan S, Hartwig D: Autologous serum eye drops for ocular surface disorders. Br J Ophthalmol. 2004;88:1467-1474.

$\checkmark 8$ Dogru M, Tsubota K: Pharmacotherapy of dry eye. Expert Opin Pharmacother. 2011;12: 325-334.

9 Kojima T, Ishida R, Dogru M, et al.: The effect of autologous serum eyedrops in the treatment of severe dry eye disease: a prospective randomized case-control study. Am J Ophthalmol. 2005;139:242-246.

10 Quinto GG, Campos M, Behrens A: Autologous serum for ocular surface diseases. Arq Bras Oftalmol. 2008 ;71(6 Suppl):47-54.

$>11$ Stenwall PA, Bergström M, Seiron P, et al.: Improving the anti-inflammatory effect of serum eye drops using allogeneic serum permissive for regulatory $\mathrm{T}$ cell induction. Acta Ophthalmol. 2015;93:654-657.

12 Tsubota K, Higuchi A: Serum application for the treatment of ocular surface disorders. Int Ophthalmol Clin. 2000;40:113-122.

13 van Setten GB, Tervo T, Viinikka L, et al.: Ocular disease leads to decreased concentrations of epidermal growth factor in the tear fluid. Curr Eye Res. 1991;10:523-527.

14 Tsubota K, Yamada M: Tear evaporation from the ocular surface. Invest Ophthalmol Vis Sci. 1992;33:2942-2950.

-15 Siervo M, Bunn D, Prado CM, et al.: Accuracy of prediction equations for serum osmolarity in frail older people with and without diabetes. Am J Clin Nutr. 2014;100:867-876.

16 Ferreira de Souza R, Kruse FE, Seitz B: [Autologous serum for otherwise therapy resistant corneal epithelial defects -- Prospective report on the first 70 eyes]. Klin Monatsbl Augenheilkd. 2001;218:720-726.

17 Chiang CC, Lin JM, Chen WL, et al.: Allogeneic serum eye drops for the treatment of severe dry eye in patients with chronic graft-versus-host disease. Cornea. 2007;26:861-863.

18 Hyon JY, Lee YJ, Yun PY: Management of ocular surface inflammation in Sjögren syndrome. Cornea. 2007;26(9 Suppl 1):S13-15

19 Kojima T, Higuchi A, Goto E, et al.: Autologous serum eye drops for the treatment of dry eye diseases. Cornea. 2008;27(Suppl 1):S25-30.

20 Matsumoto Y, Dogru M, Goto E, et al.: Autologous serum application in the treatment of neurotrophic keratopathy. Ophthalmology. 2004;111:1115-120.

21 Ogawa Y, Okamoto S, Mori T, et al.: Autologous serum eye drops for the treatment of severe dry eye in patients with chronic graft-versus-host disease. Bone Marrow Transplant. 2003;31:579-583.

22 Tananuvat N, Daniell M, Sullivan LJ, et al.: Controlled study of the use of autologous serum in dry eye patients. Cornea. 2001;20:802-806.

23 Poon AC, Geerling G, Dart JK, et al.: Autologous serum eyedrops for dry eyes and epithelial defects: clinical and in vitro toxicity studies. Br J Ophthalmol. 2001;85:1188-1197.

24 Tsubota K, Goto E, Fujita H, et al.: Treatment of dry eye by autologous serum application in Sjögren's syndrome. Br J Ophthalmol. 1999;83: 390-395

25 EN ISO 14155:2011 Clinical investigation of medical devices for human use - Good clinical practice (ISO 14155:2011), 2011.

26 EN ISO 13485:2012 (ISO 13485:2003), Medical devices - Quality management systems Requirements for regulatory purposes, 2012.

27 Schiffman RM, Christianson MD, Jacobsen G, et al.: Reliability and validity of the Ocular Surface Disease Index. Arch Ophthalmol. 2000; 118:615-621.

28 Bron AJ, Evans VE, Smith JA: Grading of corneal and conjunctival staining in the context of other dry eye tests. Cornea. 2003;22:640-650.

29 Guthoff RF, Zhivov A, Stachs O: In vivo confocal microscopy, an inner vision of the cornea a major review. Clin Exp Ophthalmol. 2009;37: 100-117.
Ziegler D, Papanas N, Zhivov A, et al.; German Diabetes Study (GDS) Group: Early detection of nerve fiber loss by corneal confocal microscopy and skin biopsy in recently diagnosed type 2 diabetes. Diabetes. 2014;63:2454-263.

31 López-García JS, García-Lozano I, Rivas L, et al.: Autologous serum eye drops diluted with sodium hyaluronate: clinical and experimental comparative study. Acta Ophthalmol. 2014; 92:e22-29.

32 Mahelková G, Veselá V, Seidler Štangová P, et al.: [Tear Osmolarity in Patients with Severe Dry Eye Syndrome Before and After Autologous Serum Treatment: a Comparison with Tear Osmolarity in Healthy Volunteers]. Cesk Slov Oftalmol. 2015;71:184-188.

33 Craig JP, Nichols KK, Akpek EK, et al.: TFOS DEWS II Definition and Classification Report. Ocul Surf. 2017;15:276-283

34 Baudouin C: The pathology of dry eye. Surv Ophthalmol. 2001;45(Suppl 2):S211-20.

35 Ubels JL, MacRae SM: Vitamin A is present as retinol in the tears of humans and rabbits. Curr Eye Res. 1984;3:815-822.

-36 Tsubota K, Goto E, Shimmura S, et al.: Treatment of persistent corneal epithelial defect by autologous serum application. Ophthalmology. 1999;106:1984-1989.

37 Khaksari M, Mazzoleni LR, Ruan C, et al: Determination of water-soluble and fat-soluble vitamins in tears and blood serum of infants and parents by liquid chromatography/mass spectrometry. Exp Eye Res. 2017;155:54-63.

38 Rössner S: Further studies on serum lipoproteins in connective tissue diseases. Atherosclerosis. 1978;31:93-99.

39 Hamsten A, de Faire U, Walldius G, et al.: Plasminogen activator inhibitor in plasma: risk factor for recurrent myocardial infarction. Lancet. 1987;2:3-9.

40 Oranskiy SP, Yeliseyeva LN, Tsanaeva AV, et al: Body composition and serum levels of adiponectin, vascular endothelial growth factor, and interleukin- 6 in patients with rheumatoid arthritis. Croat Med J. 2012;53:350-356.

41 Willeke P, Schotte H, Schlüter B, et al.: Interleukin 1 beta and tumour necrosis factor alpha secreting cells are increased in the peripheral blood of patients with primary Sjögren's syndrome. Ann Rheum Dis. 2003;62:359-362.

42 Hwang J, Chung SH, Jeon S, et al.: Comparison of clinical efficacies of autologous serum eye drops in patients with primary and secondary Sjögren syndrome. Cornea. 2014;33: 663-667. 